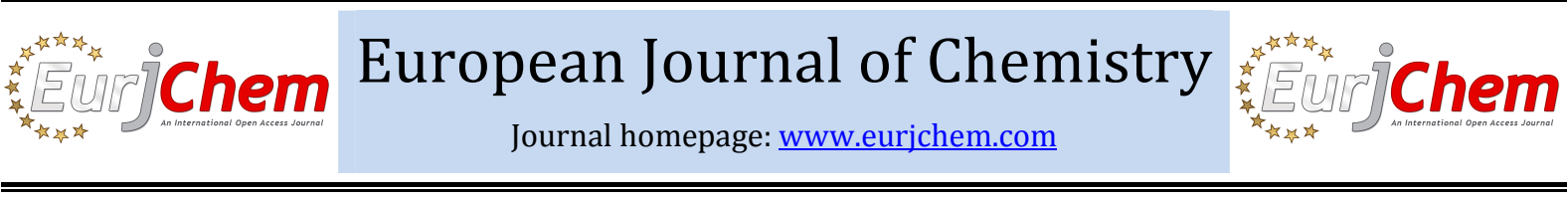

\section{Synthesis of some new pyrazolylfuropyrimidinethiones and triazolofuropyrimidinethiones}

\author{
Mohamed Abdel Megid Abdel-Hamid, Azza Mohamed Elkazak*, \\ Magdy Hamed Sead and Osama Farouk Mohamed \\ Department of Chemistry, Faculty of Education, Ain Shams University, Roxy, 11711, Cairo, Egypt \\ *Corresponding author at: Department of Chemistry, Faculty of Education, Ain Shams University, Roxy, 11711, Cairo, Egypt. Tel.: +2.0100.6422648; \\ fax:+2.02.2581243. E-mail address: az_azelkazak@yahoo.com (A.M. Elkazak).
}

\section{ARTICLE INFORMATION}

Received: 29 February 2012

Received in revised form:05 April 2012

Accepted: 15 April 2012

Online: 30 September 2012

\section{KEYWORDS}

\section{Triazoles}

Pyrazoles

Tetrazoles

Enaminones

Ditetrazoles

Furopyrimidines

\section{Introduction}

Polysubstituted furans are important heterocyclic molecules due to practical utility as a recurring unit in many natural and medicinal molecules. Many of the naturally occurring furans have shown interesting biological activities. On the other hand, pyrimidines, being integral part of DNA and RNA, exhibit diverse pharmacological properties such as effective bactericides, fungicides, viricides, insecticides and posses significant therapeutic values [1]. Motivated by these facts, the present investigation deals with combination of furan and pyrimidine moieties in a molecular framework. Furopyrimidine derivatives are an important class of heterocyclic compounds in pharmaceutical discovery research $[2,3]$. They showed antifungal [4], antifolate [5], antibacterial [6], antitumor [7], antiviral [8], and anti HCMV [9] (antihuman cytomegalovirus). As a part of our program directed to develop the chemistry of enaminones [10], and pyrimidines [11-16], the present investigation deals with the use of 2-amino-4,5diphenylfuran-3-carbonitrile (1) [17], as a starting material for the synthesis of the target pyrazolylfuropyrimidinethiones and furotriazolopyrimidinethiones.

\section{Experimental}

\subsection{Instrumentation}

All melting points were measured on Stuart SMP-3 and were uncorrected. The IR spectra were recorded on FT-IR Jasco 4000 spectrophotometer using $\mathrm{KBr}$ wafer technique. ${ }^{1} \mathrm{H}$ NMR spectra were measured on Varian Gemini spectrophotometer $200 \mathrm{MHz}$ and Varian Mercury VX-300 NMR spectrometer using TMS $(\delta \mathrm{ppm})$ as an internal standard. ${ }^{13} \mathrm{C}$ NMR spectra were measured at $75.00 \mathrm{MHz}$ in dimethylsulphoxide (DMSO- $d_{6}$ ). Mass spectra were obtained using GC-MS Shimadzu Qp-2010, GAS-chromatograph (GC-14A) and JEOL-JMS-AX500 mass spectrometer. 2-Amino-4,5-diphenylfuran-3-carbonitrile (1) was prepared according to the reported method [17].

\subsection{Synthesis}

\subsubsection{5,6-Diphenylfuro [2,3-d] pyrimidine-2,4(1H,3H)-} dithione (2)

A mixture of 1 ( $0.01 \mathrm{~mol}, 2.6 \mathrm{~g})$ and carbon disulphide $(0.02$ mol, $1.2 \mathrm{~mL})$ in ethanolic potassium hydroxide $(0.56 \mathrm{~g}$ in 20 $\mathrm{mL}$ ) was heated under reflux for $3 \mathrm{~h}$. The reaction mixture was cooled; the solid salt was dissolved in water and acidified with few drops of concentrated hydrochloric acid .The solid obtained was filtered off and recrystallized from DMF to give compound 2 as yellow crystals (Scheme 1). Yield: 72\%. M.p.: 276-278 ${ }^{\circ} \mathrm{C}$. FT-IR $\left(\mathrm{KBr}, \mathrm{cm}^{-1}\right): 3345,3200(2 \mathrm{NH}), 3054$ ( $\left.\mathrm{CH}_{\text {aromatic }}\right), 1594-1509$ ( $\mathrm{C}=\mathrm{N}$ and $\left.\mathrm{C}=\mathrm{C}\right), 1243,1206$ (2 $\left.\mathrm{C}=\mathrm{S}\right)$, 1072 (C-0-). MS (EI, $m / z,(\%)): 336$ (M, 88), 277 (30), 259 (2), 218 (3), 178 (5), 118 (5), 77 (100), 59 (9). Anal. calcd. for $\mathrm{C}_{18} \mathrm{H}_{12} \mathrm{~N}_{2} \mathrm{OS}_{2}$ : C, 64.26; H, 3.60; N, 8.33; S, 19.06. Found: C, 64.30; H, 3.70; N, 8.40; S, 19.10\%.

\subsubsection{4-Hydrazino-5,6-diphenylfuro[2,3-d]pyrimidin-2(1H)- thione (3)}

A mixture of $2(0.01 \mathrm{~mol}, 3.36 \mathrm{~g})$ and hydrazine hydrate $(0.01 \mathrm{~mol}, 0.5 \mathrm{~mL})$ in absolute ethanol $(20 \mathrm{~mL})$ was heated under reflux for $2 \mathrm{~h}$. The solid obtained was filtered off and recrystallized from ethanol to give compound $\mathbf{3}$ as yellow crystals (Scheme 1). Yield: $74 \%$. M.p.: $215-217^{\circ} \mathrm{C}$. 


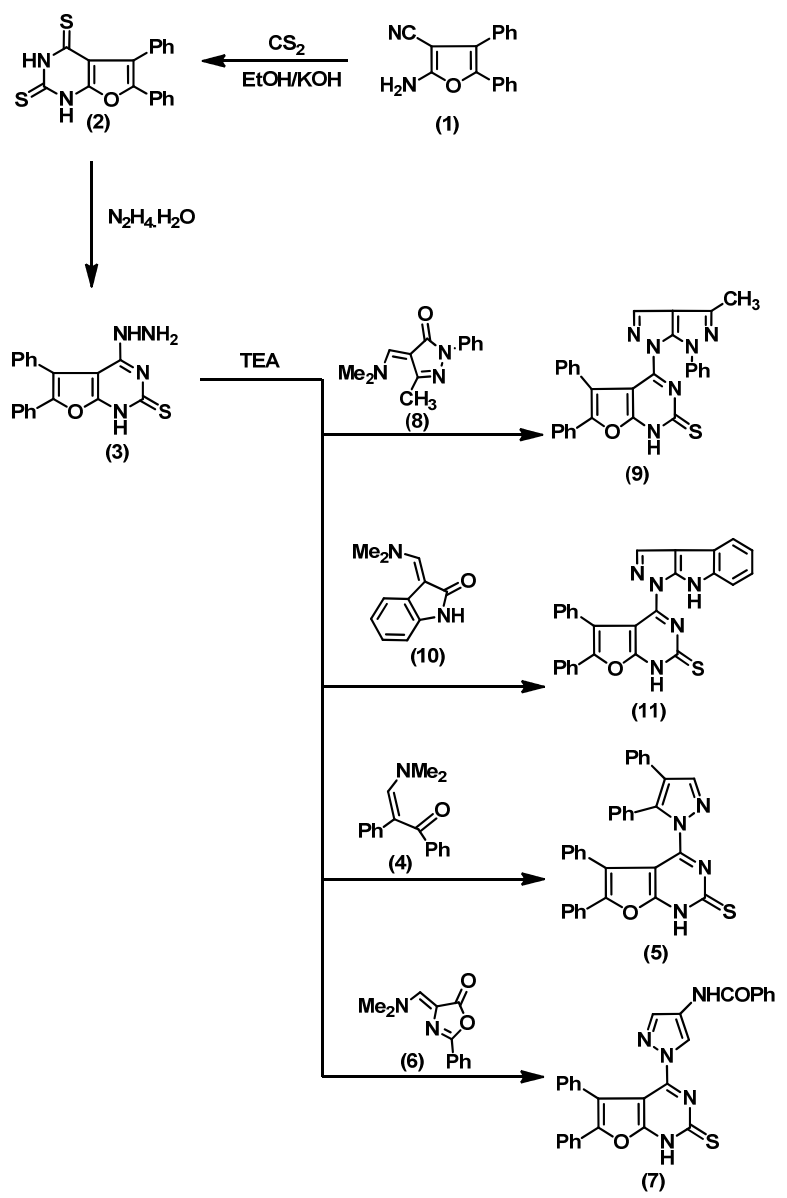

Scheme 1

FT-IR $\left(\mathrm{KBr}, \mathrm{cm}^{-1}\right)$ : 3590-3158 $\left(\mathrm{NH}_{2}, 2 \mathrm{NH}\right), 3052\left(\mathrm{CH}_{\text {arom. }}\right)$, 1567-1504 ( $\mathrm{C}=\mathrm{N}$ and $\mathrm{C}=\mathrm{C}$ ), 1217 ( $\mathrm{C}=\mathrm{S}), 1098$ (C-O-). ${ }^{1} \mathrm{H}$ NMR (200 MHz, DMSO-d6, $\delta$, ppm): 6.17 (br.s, $2 \mathrm{H}, \mathrm{NH}_{2}$ exchangeable with $\left.\mathrm{D}_{2} \mathrm{O}\right), 6.49\left(\mathrm{~s},{ }^{1} \mathrm{H}, \mathrm{N} H_{\text {hydrazine}}\right), 6.90-8.06(\mathrm{~m}, 10 \mathrm{H}, \mathrm{Ar}-H)$, $9.86\left(\mathrm{~s}, 1 \mathrm{H}, \mathrm{N} H_{\text {pyrimidine). }} \mathrm{MS}\right.$ (EI, $\left.m / z,(\%)\right): 334$ (M, 28), 336 (21), 304 (7), 229 (55), 101 (21), 77 (100). Anal. calcd. for $\mathrm{C}_{18} \mathrm{H}_{14} \mathrm{~N}_{4} \mathrm{OS}$ : C, 64.65; H, 4.22; N, 16.75; S, 9.59. Found: C, 64.80; H, $4.30 ; \mathrm{N}, 16.60 ; \mathrm{S}, 9.70 \%$.

\subsubsection{4-(4,5-Diphenyl-1H-pyrazol-1-yl)-5,6-diphenylfuro [2,3-d]pyrimidine-2(1H)-thione (5)}

A mixture of 3 (0.01 mol, 3.34 g) and 3-dimethylamino-1,2diphenylpropen-1-one $(0.01 \mathrm{~mol}, 2.51 \mathrm{~g})$ in absolute ethanol $(20 \mathrm{~mL})$ containing few drops of triethylamine was heated under reflux for $3 \mathrm{~h}$. After cooling, the solid product so formed, was collected and recrystallized from ethanol to give compound 5 as brown crystals (Scheme 1). Yield: 71\%. M.p.: 167-169 ${ }^{\circ} \mathrm{C}$. FT-IR (KBr, cm$\left.{ }^{-1}\right): 3418(\mathrm{NH}), 3055\left(\mathrm{CH}_{\text {arom. }}\right)$, 1593$1545(\mathrm{C}=\mathrm{N}$ and $\mathrm{C}=\mathrm{C}), 1185$ (C=S), 1069 (C-O-). MS (EI, $m / z$, (\%)): 522 (M, 17), 520 (100), 505 (58), 494 (2), 490 (40), 478 (1), 317 (24), 231 (16), 214 (12), 205 (7), 154 (11), 105 (27). Anal. calcd. for $\mathrm{C}_{33} \mathrm{H}_{22} \mathrm{~N}_{4} \mathrm{OS}$ : C, 75.84; H, 4.24; N, 10.72; S, 6.14. Found: $\mathrm{C}, 75.90 ; \mathrm{H}, 4.20 ; \mathrm{N}, 10.60 ; \mathrm{S}, 6.20 \%$.

\subsubsection{4-(4-Benzoylamino-1H-pyrazol-1-yl)-5,6-diphenylfuro [2,3-d]pyrimidine-2(1H)-thione (7)}

A mixture of $3 \quad(0.01 \quad \mathrm{~mol}, \quad 3.34 \quad \mathrm{~g})$ and 4 methylidenedimethylamino-2-phenyl-1,3-oxazol-5-one $\quad(0.01$ mol, $2.16 \mathrm{~g})$ in absolute ethanol $(20 \mathrm{~mL})$ containing few drops of triethylamine was heated under reflux for $3 \mathrm{~h}$. After cooling, the solid product so formed was collected by filtration and recrystallized from ethanol to give compound $\mathbf{7}$ as yellow crystals (Scheme 1). Yield: 74\%. M.p.: 222-224 ${ }^{\circ} \mathrm{C}$. FT-IR (KBr, $\left.\mathrm{cm}^{-1}\right)$ : 3421, $3276(2 \mathrm{NH}), 3061$ ( $\left.\mathrm{CH}_{\text {arom. }}\right), 1672(\mathrm{C}=0), 1615$ $1577(\mathrm{C}=\mathrm{N}$ and $\mathrm{C}=\mathrm{C}), 1231$ (C=S), 1094 (C-O-). ${ }^{1} \mathrm{H}$ NMR $(200$ MHz, DMSO- $d_{6}, \delta, \mathrm{ppm}$ ): 5.58 (s, $\left.1 \mathrm{H}, H-5_{\text {pyrazole }}\right), 7.21-7.90(\mathrm{~m}$, $15 \mathrm{H}, \mathrm{Ar}-H), 7.96(\mathrm{~s}, 1 \mathrm{H}, H-3$ pyrazole $), 8.08$ (s, $1 \mathrm{H}, \mathrm{N} H_{\text {pyrimidine }}$ exchangeable with $\left.\mathrm{D}_{2} \mathrm{O}\right), 10.38\left(\mathrm{~s}, 1 \mathrm{H}, \mathrm{N} H_{\text {amide }}\right.$ exchangeable with $\left.\mathrm{D}_{2} \mathrm{O}\right)$. MS (EI, $\left.m / z,(\%)\right): 487(\mathrm{M}-2,3), 383$ (2), $274(4)$, 271 (4), 218 (4), 215 (3), 154 (3), 120 (7), 105 (25), 77 (63), 50 (100). Anal. calcd. for $\mathrm{C}_{28} \mathrm{H}_{19} \mathrm{~N}_{5} \mathrm{O}_{2} \mathrm{~S}: \mathrm{C}, 68.70 ; \mathrm{H}, 3.91 ; \mathrm{N}, 14.31 ; \mathrm{S}$, 6.55. Found: C, 68.60; H, 3.80; N, 14.30; S, 6.60\%.

\subsubsection{5,6-Diphenyl-4-(4-methyl-6-phenylpyrazolo[3,4-c] pyrazol-1(6H)-yl)-3,4-dihydro-furo[2,3-d]pyrimidine-2(1H)- thione (9)}

A mixture of $3 \quad(0.01 \quad \mathrm{~mol}, \quad 3.34 \quad \mathrm{~g})$ and 4methylidenedimethylamino-1-phenylpyrazol-5-one $(0.01 \mathrm{~mol}$, $2.29 \mathrm{~g})$ in absolute ethanol $(20 \mathrm{~mL})$ containing few drops of triethylamine was heated under reflux for $3 \mathrm{~h}$. After cooling, the solid product so formed was filtered off and recrystallized from ethanol to give compound 9 as yellow crystals (Scheme 1). Yield: $64 \%$. M.p.: $232-234{ }^{\circ} \mathrm{C}$. FT-IR $\left(\mathrm{KBr}, \mathrm{cm}^{-1}\right): 3276(\mathrm{NH})$, 3055 ( $\mathrm{CH}_{\text {arom. }}$ ), 2969 ( $\left.\mathrm{CH}_{\text {aliph. }}\right), 1599-1544$ ( $\mathrm{C}=\mathrm{N}$ and $\left.\mathrm{C}=\mathrm{C}\right), 1262$ $(\mathrm{C}=\mathrm{S}), 1079$ (C-0-). 


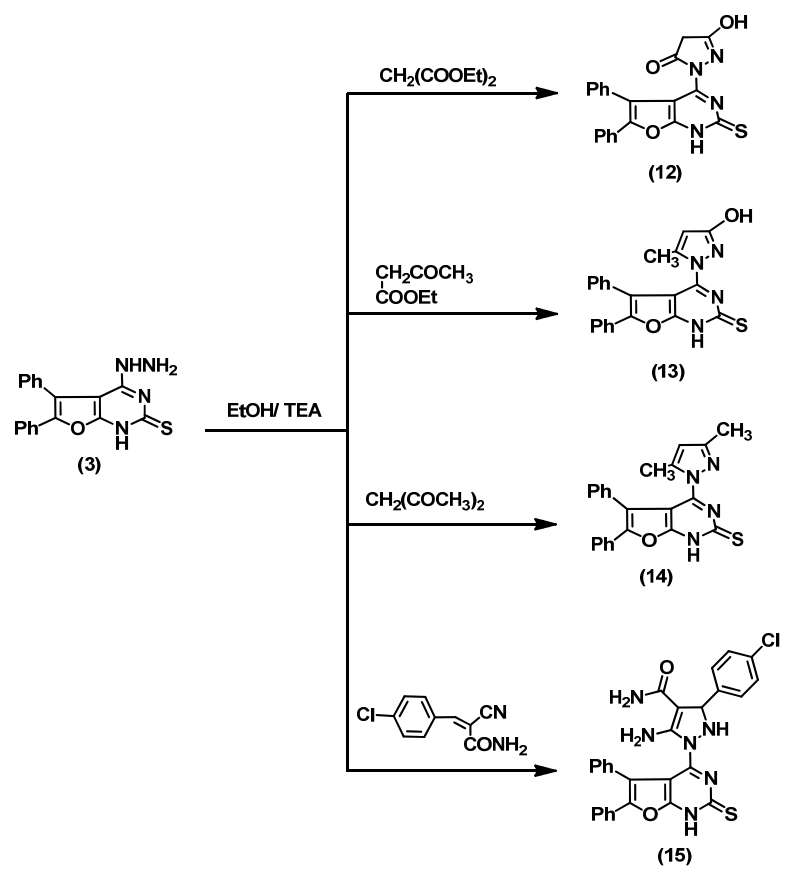

Scheme 2

MS (EI, $m / z,(\%)): 481$ (M-19, 6), 344 (11), 322 (6), 317 (3), 218 (3), 185 (100), 183 (14), 156 (16), 77 (33). Anal. calcd. for $\mathrm{C}_{29} \mathrm{H}_{20} \mathrm{~N}_{6} \mathrm{OS}: \mathrm{C}, 69.58 ; \mathrm{H}, 4.03 ; \mathrm{N}, 16.79 ; \mathrm{S}, 6.41$. Found: C, 69.60; $\mathrm{H}, 4.10 ; \mathrm{N}, 16.70 ; \mathrm{S}, 6.30 \%$.

2.2.6. 5,6-Diphenyl-4-(pyrazolo[3,4-b]indol-1-yl)-furo[2,3-d] pyrimidine-2(1H)-thione (11)

A mixture of $3(0.01 \mathrm{~mol}, 3.34 \mathrm{~g})$ and 3 methylidenedimethylaminoindol-2(1H)-one $(0.01 \mathrm{~mol}, 1.88 \mathrm{~g})$ in absolute ethanol $(20 \mathrm{~mL})$ containing few drops of triethylamine was heated under reflux for $3 \mathrm{~h}$. After cooling, the solid product so formed was filtered off and recrystallized from ethanol to give compound $\mathbf{1 1}$ as brown crystals (Scheme 1). Yield: 76\%. M.p.: 238-240 ${ }^{\circ} \mathrm{C}$. FT-IR (KBr, $\left.\mathrm{cm}^{-1}\right): 3400,3312$ (2 $\mathrm{NH}), 3055$ ( $\left.\mathrm{CH}_{\text {arom. }}\right), 1594-1541(\mathrm{C}=\mathrm{N}$ and $\mathrm{C}=\mathrm{C}), 1238(\mathrm{C}=\mathrm{S})$, 1137 (C-0-). ${ }^{1} \mathrm{H}$ NMR (300 MHz, DMSO- $\left.d_{6}, \delta, \mathrm{ppm}\right): 7.19-7.57$ $(\mathrm{m}, 14 \mathrm{H}, \mathrm{Ar}-H), 7.96\left(\mathrm{~s}, 1 \mathrm{H}, H-3_{\text {pyrazole }}\right), 8.64\left(\mathrm{~s}, 1 \mathrm{H}, \mathrm{N} H_{\text {indole }}\right.$ exchangeable with $\left.\mathrm{D}_{2} \mathrm{O}\right), 10.95\left(\mathrm{~s}, 1 \mathrm{H}, \mathrm{N} H_{\text {pyrimidine }}\right.$ exchangeable with $\left.\mathrm{D}_{2} \mathrm{O}\right)$. MS (EI, $\left.m / z,(\%)\right): 460(\mathrm{M}+1,1), 344(2), 305$ (2), 265 (1), 241 (7), 218 (7), 203 (100), 194 (8), 178 (15), 105 (31\%). Anal. calcd. for $\mathrm{C}_{27} \mathrm{H}_{17} \mathrm{~N}_{5} \mathrm{OS}$ : C, 70.57; H, 3.73; N, 15.24; S, 6.98. Found: C, 70.50; H, 3.80; N, 15.30; S, 6.90\%.

\subsubsection{5,6-Diphenyl-4-(3-hydroxy-5-oxo-1H,4H-pyrazol-1-yl)-} furo[2,3-d]pyrimidine-2(1H)-thione (12)

A mixture of $3(0.01 \mathrm{~mol}, 3.34 \mathrm{~g})$ and diethyl malonate $(0.01 \mathrm{~mol}, 1.5 \mathrm{~mL})$ in absolute ethanol $(20 \mathrm{~mL})$ containing few drops of triethylamine was heated under reflux for $3 \mathrm{~h}$. After cooling, the solid obtained was filtered off and recrystallized from DMF to give compound $\mathbf{1 2}$ as white crystals (Scheme 2). Yield: 74\%. M.p.: 244-246 ${ }^{\circ}$ C. FT-IR (KBr, cm$\left.{ }^{-1}\right): 3441(\mathrm{OH})$, $3298(\mathrm{NH}), 3063$ ( $\left.\mathrm{CH}_{\text {aromatic }}\right), 2924\left(\mathrm{CH}_{\text {aliphatic }}\right), 1666(\mathrm{C}=0)$, 1620-1573 (C=N and $\mathrm{C}=\mathrm{C}), 1230$ (C=S), 1099 (C-0-). ${ }^{1} \mathrm{H}$ NMR (300 MHz, DMSO- $\left.d_{6}, \delta, \mathrm{ppm}\right): 5.56\left(\mathrm{~s}, 2 \mathrm{H}, \mathrm{CH}_{2}\right), 7.09-7.37(\mathrm{~m}$, $10 \mathrm{H}, \mathrm{Ar}-H$ ) , $8.05\left(\mathrm{~s}, 1 \mathrm{H}, \mathrm{N} H\right.$ exchangeable with $\left.\mathrm{D}_{2} \mathrm{O}\right), 10.34$ (s, $1 \mathrm{H}, \mathrm{OH}$ exchangeable with $\left.\mathrm{D}_{2} \mathrm{O}\right) .{ }^{13} \mathrm{C}$ NMR $\left(75 \mathrm{MHz}\right.$, DMSO- $d_{6}, \delta$, ppm): $34.5\left(\mathrm{CH}_{2}\right), 82.5$ (bridged $\mathrm{C}_{3}$-furan), 125.5, 127.2, 128.3(2), 128.4 (2) (corresponding to six phenyl carbons), 131
( $\mathrm{C}_{4}$-furan), 132.1, 136.3(corresponding to two phenyl carbons), 143.4 (bridged $\mathrm{C}_{2}$-furan), 153.8 (C5-furan), $162.3 \quad$ (2) (corresponding to $\mathrm{C}-\mathrm{OH}$, pyrazole and $\mathrm{C}=\mathrm{N}$, pyrimidine), 162.7 $(\mathrm{C}=0)$ and $168.6(\mathrm{C}=\mathrm{S})$. MS (EI, $m / z,(\%)): 402(\mathrm{M}, 1), 404(1)$, 325 (1), 307 (1), 230 (1), 178 (1), $154\left(2 \mathrm{C}_{6} \mathrm{H}_{5}, 1\right) 105$ (3), 77(100). Anal. calcd. for $\mathrm{C}_{21} \mathrm{H}_{14} \mathrm{~N}_{4} \mathrm{O}_{3} \mathrm{~S}$ : C, 62.68; $\mathrm{H}, 3.51 ; \mathrm{N}$, 13.92; S, 7.97. Found: C, 62.80; H, 3.40; N, 13.80; S, 7.90.

2.2.8. 5,6-Diphenyl-4-(3-hydroxy-5-methyl-1H-pyrazol-1-yl)furo[2,3-d]pyrimidine-2(1H)-thione (13)

A mixture of $3(0.01 \mathrm{~mol}, 3.34 \mathrm{~g})$ and ethyl acetoacetate $(0.01 \mathrm{~mol}, 1.3 \mathrm{~mL})$ in absolute ethanol $(20 \mathrm{~mL})$ containing few drops of triethylamine was heated under reflux for $3 \mathrm{~h}$. After cooling, the solid obtained was filtered off and recrystallized from dioxan to give compound $\mathbf{1 3}$ as brown crystals (Scheme 2). Yield: $64 \%$. M.p.: $264-266^{\circ} \mathrm{C}$. FT-IR (KBr, cm-1): $3600-3200$ $(\mathrm{OH}, \mathrm{NH}), 3059\left(\mathrm{CH}_{\text {arom. }}\right), 2978\left(\mathrm{CH}_{\text {aliph. }}\right), 1602-1570(\mathrm{C}=\mathrm{N}$ and $\mathrm{C}=\mathrm{C}$ ), 1261 (C=S), 1068 (C-O-). MS (EI, $\mathrm{m} / \mathrm{z},(\%)): 400$ (M, 1), 384 (1), 372 (1), 366 (1), 356 (1), 323 (1), 246 (3), 154 ( 1), 77 (6), 61 (100), 42 (15). Anal. calcd. for $\mathrm{C}_{22} \mathrm{H}_{16} \mathrm{~N}_{4} \mathrm{O}_{2} \mathrm{~S}$ : C, 65.99; $\mathrm{H}$, 4.03; N, 13.99; S, 8.01. Found: C, 65.90; H, 4.10; N, 13.90; S, $8.10 \%$.

\subsubsection{4-(3,5-Dimethyl-1H-pyrazol-1-yl)-5,6-diphenylfuro} [2,3-d]pyrimidine-2(1H)-thione (14)

A mixture of $3(0.01 \mathrm{~mol}, 3.34 \mathrm{~g})$ and acetylacetone $(0.01$ $\mathrm{mol}, 1 \mathrm{~mL})$ in absolute ethanol $(20 \mathrm{~mL})$ containing few drops of triethylamine was heated under reflux for $3 \mathrm{~h}$. After cooling, the solid obtained was filtered off and recrystallized from ethanol to give 14 as yellow crystals (Scheme 2). Yield: 74\%. M.p.: 121$123{ }^{\circ} \mathrm{C}$. FT-IR (KBr, cm-1): $3251(\mathrm{NH}), 3059$ ( $\left.\mathrm{CH}_{\text {arom. }}\right), 2974$ ( $\mathrm{CH}_{\text {aliph.) }}$ ), 1589-1551 ( $\mathrm{C}=\mathrm{N}$ and $\left.\mathrm{C}=\mathrm{C}\right), 1250(\mathrm{C}=\mathrm{S}), 1057$ (C- O-). ${ }^{1} \mathrm{H}$ NMR $\left(200 \mathrm{MHz}\right.$, DMSO- $\left.d_{6}, \delta, \mathrm{ppm}\right): 3.34\left(\mathrm{~s}, 3 \mathrm{H}, \mathrm{CH}_{3}-\mathrm{C}_{5}\right.$ pyrazole), $4.27\left(\mathrm{~s}, 3 \mathrm{H}, \mathrm{CH}_{3}-\mathrm{C}_{3}\right.$ pyrazole), $6.54(\mathrm{~s}, 1 \mathrm{H}, \mathrm{CH}$ pyrazole), 7.15-7.73 (m, 10H, Ar-H), $11.98(\mathrm{~s}, 1 \mathrm{H}, \mathrm{NH})$. MS (EI, $m / z,(\%)): 396$ (M-2, 10), 370 (2), 358 (7), 357 (7), 321 (4), 292 (9), 244 (10), 154 (7), 106 (14), 77 (55), 59 (3), 43 (100). 


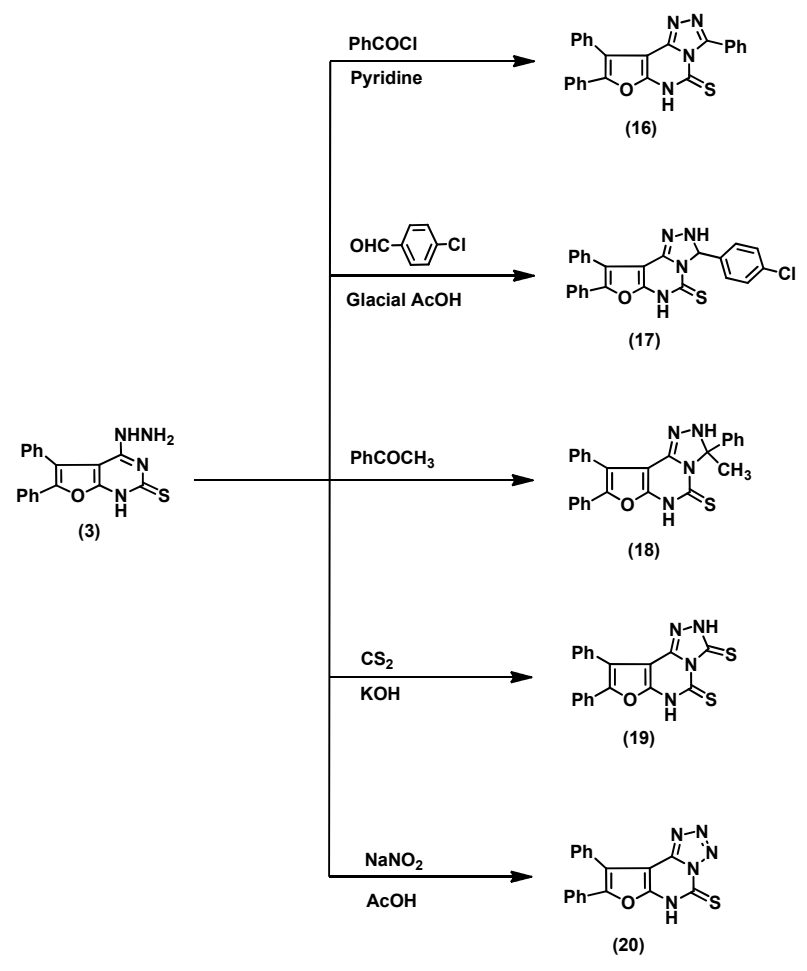

Scheme 3

Anal. calcd. for $\mathrm{C}_{23} \mathrm{H}_{18} \mathrm{~N}_{4} \mathrm{OS}$ : C, 69.33; H, 4.55; N, 14.06; S, 8.05. Found: C, $69.40 ; \mathrm{H}, 4.60 ; \mathrm{N}, 14.10 ; \mathrm{S}, 8.10 \%$.

\subsubsection{4-[5-Amino-4-carboxamido-3-(4-chlorophenyl)-1H- pyrazol-1-yl]-5,6-diphenyl-furo[2,3-d]pyrimidine-2(1H)- thione (15)}

A mixture of $3(0.01 \mathrm{~mol}, 3.34 \mathrm{~g})$ and $p$-chlorobenzlidenecyanoacetamide $(0.01 \mathrm{~mol}, 2 \mathrm{~g})$ in absolute ethanol $(20 \mathrm{~mL})$ containing few drops of triethylamine was heated under reflux for $3 \mathrm{~h}$. After cooling, the solid obtained was filtered off and recrystallized from ethanol to give compound $\mathbf{1 5}$ as red crystals (Scheme 2). Yield: 74\%. M.p.: $235-237{ }^{\circ} \mathrm{C}$. FT-IR (KBr, $\left.\mathrm{cm}^{-1}\right)$ : 3600-3200 (2NH, 2NH 2$), 3059$ ( $\left.\mathrm{CH}_{\text {arom. }}\right), 2924\left(\mathrm{CH}_{\text {aliph. }}\right)$, $1639(\mathrm{C}=0), 1593-1554(\mathrm{C}=\mathrm{N}$ and $\mathrm{C}=\mathrm{C}), 1219(\mathrm{C}=\mathrm{S}), 1092(\mathrm{C}-\mathrm{O}-$ ). MS (EI, $m / z,(\%)): 540$ (M, 11), 112 (4), 106 (5), 77 (14), 61 (100), 44 (76). Anal. calcd. for $\mathrm{C}_{28} \mathrm{H}_{21} \mathrm{~N}_{6} \mathrm{O}_{2} \mathrm{SCl}$ : C, 62.16; $\mathrm{H}, 3.91$; $\mathrm{N}, 15.53 ; \mathrm{S}, 5.93 ; \mathrm{Cl}, 6.55$. Found: $\mathrm{C}, 62.20 ; \mathrm{H}, 3.80 ; \mathrm{N}, 15.50 ; \mathrm{S}$, $6.00 ; \mathrm{Cl}, 6.60 \%$.

\subsubsection{3,8,9-Triphenylfuro[2',3`4,5]pyrimido[6,1-c]-1,2,4- triazole-5(6H)-thione (16)}

A mixture of $3(0.01 \mathrm{~mol}, 3.34 \mathrm{~g})$ and benzoyl chloride $(0.01$ mol $1.2 \mathrm{~mL})$ in pyridine $(20 \mathrm{~mL})$ was heated on boiling water bath for $3 \mathrm{~h}$. The mixture was left to cool at room temperature, and then poured onto crushed ice containing $\mathrm{HCl}$, the solid obtained was filtered off and recrystallized from benzene to give compound 16 as white crystals (Scheme 3). Yield: 81\%. M.p.: $132-134^{\circ} \mathrm{C}$. FT-IR $\left(\mathrm{KBr}, \mathrm{cm}^{-1}\right): 3354(\mathrm{NH}), 3065$ ( $\left.\mathrm{CH}_{\text {arom. }}\right)$, 1591-1503 ( $\mathrm{C}=\mathrm{N}$ and $\mathrm{C}=\mathrm{C}), 1205(\mathrm{C}=\mathrm{S}), 1065$ (C-O-). MS (EI, $m / z,(\%)): 422(\mathrm{M}+2,45), 345$ (25), 336 (40), 286 (42), 167 (70), 162 (57), 79.(85), 77 (84), 69 (100). Anal. calcd. for $\mathrm{C}_{25} \mathrm{H}_{16} \mathrm{~N}_{4} \mathrm{OS}$ : C, 71.41; H, 3.84; N, 13.32; S, 7.63. Found: C, 71.50; $\mathrm{H}, 3.80 ; \mathrm{N}, 13.40 ; \mathrm{S}, 7.50 \%$.
2.2.12. 3-(4-Chlorophenyl)-2,3-dihydro-8,9-diphenylfuro $[2 \nmid 3: 4,5]$ pyrimido[6,1-c]-1,2,4-triazole-5(6H)-thione (17)

A mixture of $3(0.01 \mathrm{~mol}, 3.34 \mathrm{~g})$ and $p$-chlorobenzaldehyde $(0.01 \mathrm{~mol}, 1.41 \mathrm{~g})$ in glacial acetic acid $(20 \mathrm{~mL})$ was heated under reflux for $2 \mathrm{~h}$. After cooling, the solid obtained was filtered off and recrystallized from ethanol to give compound 17 as red crystals (Scheme 3). Yield: $85 \%$. M.p.: $>300^{\circ}$ C. FT-IR $\left(\mathrm{KBr}, \mathrm{cm}^{-1}\right): 3424,3329(2 \mathrm{NH}), 3025$ ( $\left.\mathrm{CH}_{\text {arom. }}\right), 1613-1544$ (C=N and $\mathrm{C}=\mathrm{C}), 1213$ (C=S), 1086 (C-0-). ${ }^{1} \mathrm{H}$ NMR (200 MHz, DMSO$\left.d_{6}, \delta, \mathrm{ppm}\right): 6.38\left(\mathrm{~s}, 1 \mathrm{H}, \mathrm{C}_{3}-H\right), 7.52-7.58(\mathrm{~m}, 14 \mathrm{H}, \mathrm{Ar}-\mathrm{H}), 8.29(\mathrm{~s}$, $1 \mathrm{H}, \mathrm{N} H$, triazole exchangeable with $\left.\mathrm{D}_{2} \mathrm{O}\right), 10.80(\mathrm{~s}, 1 \mathrm{H}, \mathrm{NH}$, pyrimidine exchangeable with $\mathrm{D}_{2} \mathrm{O}$ ). MS (EI, $\mathrm{m} / z,(\%)$ ): 459 (M+2, 27), 345 (49), 303 (27), 105 (85), 77 (72), 51 (100). Anal. calcd. for $\mathrm{C}_{25} \mathrm{H}_{17} \mathrm{~N}_{4} \mathrm{OSCl}$ : C, 65.71; H, 3.75; N, 12.26; $\mathrm{S}, 7.02 ; \mathrm{Cl}$, 7.76. Found: C, $65.80 ; \mathrm{H}, 3.70 ; \mathrm{N}, 12.30 ; \mathrm{S}, 7.10 ; \mathrm{Cl}, 7.60 \%$.

\subsubsection{3-Methyl-3,8,9-triphenylfuro[2',3 $: 4,5]$ pyrimido[6,1- c]-1,2,4-triazole-5(6H)-thione (18)}

A mixture of $3(0.01 \mathrm{~mol}, 3.34 \mathrm{~g}$,$) and acetophenone (0.01$ mol, $1.2 \mathrm{~mL})$ in glacial acetic acid $(20 \mathrm{~mL})$ was heated under reflux for $3 \mathrm{~h}$. After cooling, the solid obtained was filtered off and recrystallized from ethanol to give compound $\mathbf{1 8}$ as yellow crystals (Scheme 3). Yield: 89\%. M.p.: 254-255 ${ }^{\circ} \mathrm{C}$. FT-IR (KBr, $\left.\mathrm{cm}^{-1}\right)$ : 3434, $3340(2 \mathrm{NH}), 3043\left(\mathrm{CH}_{\text {arom. }}\right), 2929\left(\mathrm{CH}_{\text {aliph. }}\right), 1578-$ 1523 (C=N and $\mathrm{C}=\mathrm{C}), 1210$ (C=S), 1073 (C-O-). ${ }^{1} \mathrm{H}$ NMR (200 MHz, DMSO- $\left.d_{6}, \delta, \mathrm{ppm}\right): 2.12\left(\mathrm{~s}, 3 \mathrm{H}, \mathrm{CH}_{3}\right), 7.38-7.8(\mathrm{~m}, 15 \mathrm{H}, \mathrm{Ar}-$ $H$ ), 9.32 (s, $1 \mathrm{H}, \mathrm{N} H$ triazole exchangeable with $\mathrm{D}_{2} \mathrm{O}$ ), 10.90 (s, $1 \mathrm{H}, \mathrm{N} H$ pyrimidine exchangeable with $\left.\mathrm{D}_{2} \mathrm{O}\right)$. MS (EI, $\left.m / z,(\%)\right)$ : 435 (M-1, 1), 421 (1), 344 (1), 282 (2), 103 (100), 77 (99.9). Anal. calcd. for $\mathrm{C}_{26} \mathrm{H}_{20} \mathrm{~N}_{4} \mathrm{OS}$ : C, 71.54; H, 4.62; N, 12.83; S, 7.35 . Found: C, 71.40; H, 4.70; N, 12.70; S, 7.40\%. 


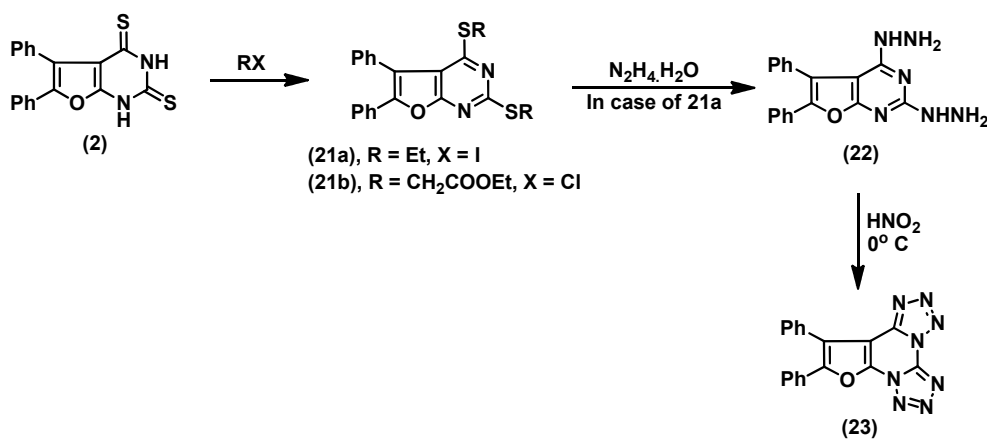

Scheme 4

\subsubsection{8,9-Diphenylfuro[2;3 :4,5]pyrimido[6,1-c]-1,2,4- triazole-3,5(2H,6H)-dithione (19)}

A mixture of $3(0.01 \mathrm{~mol}, 3.34 \mathrm{~g})$ carbon disulphide $(0.01$ $\mathrm{mol}, 0.6 \mathrm{~mL})$ and potassium hydroxide $(0.01 \mathrm{~mol}, 0.56 \mathrm{~g})$ in ethanol $(20 \mathrm{~mL})$ was heated under reflux for $3 \mathrm{~h}$. After cooling, the solid obtained upon neutralization with dilute $\mathrm{HCl}$ was filtered off and recrystallized from dioxan to give compound 19 as yellow crystals (Scheme 3). Yield: 72\%. M.p.: $256-258^{\circ} \mathrm{C}$. FTIR (KBr, cm$\left.{ }^{-1}\right)$ : 3396, 3210 (2NH), 3059 (CHarom.), 1596-1511 ( $\mathrm{C}=\mathrm{N}$ and $\mathrm{C}=\mathrm{C}), 1213,1165$ (2C=S), 1066 (C-0-). MS (EI, $\mathrm{m} / \mathrm{z}$, (\%)): 360 (M-0, 13), 359 (20), 219 (20), 218 (17), 77 (47), 76 (100). Anal. calcd. for $\mathrm{C}_{19} \mathrm{H}_{12} \mathrm{~N}_{4} \mathrm{OS}_{2}$ : C, 60.62; $\mathrm{H}, 3.21 ; \mathrm{N}, 14.88 ; \mathrm{S}$, 17.04. Found: C, $60.70 ; \mathrm{H}, 3.20$; N, $14.90 ; \mathrm{S}, 16.90 \%$.

\subsubsection{8,9-Diphenylfuro[2 3 , $: 4,5]$ pyrimido[1,6-d]-1,2,3,4- tetrazole-5(6H)-thione (20)}

A mixture of 3 (0.01 mol, $3.34 \mathrm{~g})$ and sodium nitrite $(0.01$ mol, $0.69 \mathrm{~g})$ in glacial acetic acid $(20 \mathrm{~mL})$ was stirred at room temperature for $4 \mathrm{~h}$. The solid product so formed was collected and recrystallized from ethanol to give compound $\mathbf{2 0}$ as yellow crystals (Scheme 3). Yield: $74 \%$. M.p.: $118-120^{\circ} \mathrm{C}$. FT-IR (KBr, $\left.\mathrm{cm}^{-1}\right)$ : $3390(\mathrm{NH}), 3058\left(\mathrm{CH}_{\text {arom. }}\right), 1593-1570(\mathrm{C}=\mathrm{N}$ and $\mathrm{C}=\mathrm{C})$, 1249 (C=S), 1064 (C-O-). MS (EI, $m / z,(\%)): 347$ (M+2, 2), 311 (1), 297 (2), 270 (3), 216 (5), 155 (6), 140 (6), 105 (80), 77 (100). Anal. calcd. for $\mathrm{C}_{18} \mathrm{H}_{11} \mathrm{~N}_{5} \mathrm{OS}$ : C, 62.59; H, 3.21; N, 20.28; S, 9.28. Found: C, $62.70 ; \mathrm{H}, 3.20$; N, 20.30; S, 9.20\%.

\subsubsection{2,4-Diethylthio-5,6-diphenylfuro[2,3-d]pyrimidine (21a)}

A mixture of $2(0.01 \mathrm{~mol}, 3.36 \mathrm{~g})$, ethyl iodide $(0.02 \mathrm{~mol}, 1.6$ $\mathrm{mL})$ and aqueous potassium hydroxide in DMF $(20 \mathrm{~mL})$ was heated under reflux for $3 \mathrm{~h}$. The mixture was left to cool at room temperature, and then poured onto crushed ice, the solid obtained was filtered off and recrystallized from ethanol- $\mathrm{H}_{2} \mathrm{O}$ to give compound 21a as white crystals (Scheme 4). Yield: $83 \%$. M.p.: $100-102{ }^{\circ} \mathrm{C}$. FT-IR ( $\left.\mathrm{KBr}, \mathrm{cm}^{-1}\right)$ : $3054\left(\mathrm{CH}_{\text {arom. }}\right), 2969$ ( $\left.\mathrm{CH}_{\text {aliph. }}\right), 1601-1573$ (C=N and C=C), 1058 (C-O-). ${ }^{1} \mathrm{H}$ NMR $(200$ MHz, DMSO- $\left.d_{6}, \delta, \mathrm{ppm}\right): 1.21\left(\mathrm{t}, 3 \mathrm{H}, \mathrm{CH}_{3}\right), 1.36\left(\mathrm{t}, 3 \mathrm{H}, \mathrm{CH}_{3}-\mathrm{CH}_{2}\right)$, 3.14 (q, $\left.2 \mathrm{H}, \mathrm{CH}_{2}-\mathrm{CH}_{3}\right), 3.40\left(\mathrm{q}, 2 \mathrm{H}, \mathrm{CH}_{2}\right), 7.33-7.51(\mathrm{~m}, 10 \mathrm{H}, \mathrm{Ar}-$ H). MS (EI, $m / z,(\%)): 392$ (M, 43), 393 (11), 394 (6), 377 (7), 363 (7), 331 (6), 105 (16), 77 (41), 69 (100). Anal. calcd. for $\mathrm{C}_{22} \mathrm{H}_{20} \mathrm{~N}_{2} \mathrm{OS}_{2}$ : C, 67.32; H, 5.14; N, 7.14; S, 16.34. Found: C, $67.30 ; \mathrm{H}, 5.20 ; \mathrm{N}, 7.20 ; \mathrm{S}, 16.50 \%$.

\subsubsection{2,4-Dicarbethoxymethylthio-5,6-diphenylfuro[2,3-d] pyrimidine (21b)}

A mixture of $2(0.01 \mathrm{~mol}, 3.36 \mathrm{~g})$ and ethylchloroacetate $(0.02 \mathrm{~mol}, 2.1 \mathrm{~mL})$ in DMF $(20 \mathrm{~mL})$ containing few drops of triethylamine was heated under reflux for $4 \mathrm{~h}$. The solid obtained was filtered off and recrystallized from ethanol to give 21b as white crystals (Scheme 4). Yield: $77 \%$. M.p.: $184-186{ }^{\circ} \mathrm{C}$. FT-IR (KBr, cm-1): 3052 ( $\left.\mathrm{CH}_{\text {arom. }}\right), 2987$ ( $\mathrm{CH}_{\text {aliph. }}$ ), 1742, 1674 $(2 \mathrm{C}=0), 1618-1531$ ( $\mathrm{C}=\mathrm{N}$ and $\mathrm{C}=\mathrm{C}), 1063$ (C-O-). MS (EI, $m / z$, (\%)): 508 (M, 1), 509 (1), 242 (1), 230 (1), 92 (4), 91 (100), 61 (2). Anal. calcd. for $\mathrm{C}_{26} \mathrm{H}_{24} \mathrm{~N}_{2} \mathrm{O}_{5} \mathrm{~S}_{2}$ : C, 61.39; $\mathrm{H}, 4.76$; N, 5.51; $\mathrm{S}$, 12.61. Found: C, $61.40, \mathrm{H}, 4.80$; N, 5.60; S, $12.70 \%$.

\subsubsection{2,4-Dihydrazino-5,6-diphenylfuro[2,3-d]pyrimidine} (22)

A mixture of $21 \mathrm{a}(0.01 \mathrm{~mol}, 3.92 \mathrm{~g}$ ) $)$ and hydrazine hydrate $(0.02 \mathrm{~mol}, 1 \mathrm{~mL})$ in absolute ethanol $(20 \mathrm{~mL})$ was heated under reflux for $40 \mathrm{~h}$. The solid obtained was filtered off and recrystallized from ethanol to give $\mathbf{2 2}$ as white crystals (Scheme 4). Yield: $74 \%$. M.p.: $185-187{ }^{\circ} \mathrm{C}$. FT-IR $\left(\mathrm{KBr}, \mathrm{cm}^{-1}\right)$ : 3469-3125 (2NH, 2NH$), 3054\left(\mathrm{CH}_{\text {arom. }}\right), 1579-1521(\mathrm{C}=\mathrm{N}$ and $\mathrm{C}=\mathrm{C}), 1056$ (C-O-). MS (EI, $m / z,(\%)): 318(\mathrm{M}-\mathrm{N}, 16), 152$ (24), 145 (24), 77 (92), 62 (32), 51 (100). Anal. calcd. for $\mathrm{C}_{18} \mathrm{H}_{16} \mathrm{~N}_{6} \mathrm{O}$ : C, 65.05; H, 4.85; N, 25.29. Found: C, 65.10; H, 4.90; N, 25.20\%.

\subsubsection{0,11-Diphenylfuro[2 3 ,3 $: 4,5]$ pyrimido[1,6-d:3,2-d]- 1,2,3,4-bistetrazole (23)}

A mixture of $22(0.01 \mathrm{~mol}, 3.32 \mathrm{~g})$ and sodium nitrite $(0.01$ mol, $0.69 \mathrm{~g})$ in glacial acetic acid $(20 \mathrm{~mL})$ was stirred at room temperature for $4 \mathrm{~h}$. The solid product so formed was collected and recrystallized from ethanol to give compound $\mathbf{2 3}$ as brown crystals (Scheme 4). Yield: 78\%. M.p.: $118-120^{\circ} \mathrm{C}$. FT-IR (KBr, $\left.\mathrm{cm}^{-1}\right)$ : 3055 ( $\mathrm{CH}_{\text {arom. }}$ ), 1529-1494 (C=N and C=C), 1054 (C-O-). MS (EI, $m / z$, (\%)): 354 (M, 1), 355 (1), 326 (1), 298 (1), 277 (1), 200 (4), 154 (1), 77 (100). Anal. calcd. for $\mathrm{C}_{18} \mathrm{H}_{10} \mathrm{~N}_{8} \mathrm{O}: \mathrm{C}, 61.02$; H, 2.84; N, 31.62. Found: C, 61.10; H, 2.90; N, 31.60\%.

\section{Results and discussion}

\subsection{Synthesis}

It has been reported that, the reaction of heterocyclic $o$ aminonitriles with carbon disulphide in basic medium gives fused pyrimidinethione [18]. Thus, when compound $\mathbf{1}$ was allowed to react with $\mathrm{CS}_{2}$ in alcoholic $\mathrm{KOH}$ solution, 5,6diphenylfuro[2,3- $d$ ] pyrimidine-2,4(1H,3H)-dithione (2) was obtained. IR spectrum of compound 2 revealed the absence of $\mathrm{C} \equiv \mathrm{N}$ function at $2200 \mathrm{~cm}^{-1}$ and displayed two absorption bands attributed to $2 \mathrm{C}=\mathrm{S}$ at 1243 and $1206 \mathrm{~cm}^{-1}$ and its mass spectrometry showed a molecular ion peak at $m / z 336\left(\mathrm{M}^{+}\right.$, $88 \%$ ). Compound $\mathbf{2}$ is considered as a convinent synthon for the synthesis of some novel pyrazolylfuropyrimidinethiones. Thus, the interaction of hydrazino compound 3 with some interesting enaminones like 1-dimethylamino-2,3-diphenylpropen-3-one (4) in absolute ethanol containing few drops of triethylamine led to formation of 4-(4,5-diphenylpyrazol-1-yl)- 
5,6-diphenylfuro[2,3- $d]$ pyrimidine-2(1H)-thione (5). While, the reaction of compound 3 with 4-methylidenedimethyl-amino-2phenyl-1,3-oxazol-5-one (6) [19], involved ring opening of oxazolone ring and afforded 4-(4-benzoylaminopyrazol-1-yl)5,6-diphenylfuro[2,3- $d]$ pyrimidine-2(1H)-thione (7). Moreover, polynuclear heterocyclic systems having pyrazolylfuropyrimidinethiones $\mathbf{9}$ and $\mathbf{1 1}$ were synthesized by treatment of compound 3 with 4-methylidenedimethylamino-3-methyl-1phenylpyrazol-5-one (8) [20], and 3-methylidenedimethylaminoindol-2(1H)-one (10) [21], respectively in absolute ethanol containing catalytic amount of triethylamine (Scheme 1).

On the other hand, pyrazolylfuropyrimidinethione derivatives were prepared from the reaction of the hydrazino compound $\mathbf{3}$ with 1,3-dicarbonyl compounds. Therefore, compound 3 reacted with diethyl malonate, ethyl acetoacetate and acetylacetone in absolute ethanol containing catalytic amount of triethylamine giving pyrazolylfuropyrimidinethiones (12-14). The structures of compounds 12-14 were established from their correct elemental analyses and spectroscopic data. Whereas, the 4-[5-amino-4-carboxamido-3-(4-chlorophenyl)2,3-dihydropyrazol-1-yl]-5,6-Diphenylfuro[2,3- $d$ ] pyrimidine$2(1 H)$-thione (15) was obtained from the reaction of compound 3 with $p$-chlorobenzylidenecyanoacetamide under the same reaction conditions (Scheme 2).

Also, one of our targets was the use of compound $\mathbf{3}$ to synthesize some new furotriazolopyrimidinethiones. Thus, the reaction of compound 3 with benzoyl chloride in boiling pyridine yielded 3,8,9-triphenylfuro[2`,3:4,5]pyrimido[6,1-c]1,2,4-triazole-5(6H)-thione (16). Formation of 16 involves benzoylation followed by cyclocondensation process. Condensation of compound $\mathbf{3}$ with $p$-chlorobenzaldehyde and acetophenone in boiling glacial acetic acid afforded the arylidenehydrazono intermediate, which underwent cycloaddition reaction to give 3-(4-chlorophenyl)-2,3-dihydro-8,9diphenyl-furo[2`,3 :4,5]pyrimido[6,1-c]-1,2,4-triazole-5(6H)thione (17) and 3-methyl-3,8,9-triphenylfuro[2`,3:4,5] pyrimido[6,1-c]-1,2,4-triazole-5(6H)-thione (18), respectively. Also, the effect of carbon disulphide in alcoholic potassium hydroxide and nitrous acid on compound $\mathbf{3}$ was studied and yielded furotriazolopyrimidinedithione (19) and furotetrazolopyrimidinethione (20), respectively (Scheme 3). Compounds 16-20 were established on the basis of their elemental analysis and spectral data (see experimental section).

Furthermore, alkylation of compound $\mathbf{2}$ with ethyl iodide in $\mathrm{DMF} / \mathrm{KOH}$ mixture and ethyl chloroacetate in boiling DMF yielded 2,4-dialkylthio derivatives 21a, b, respectively. Treatment of 21a with hydrazine hydrate in boiling ethanol afforded 2,4-dihydrazino-5,6-diphenylfuro[2,3-d]pyrimidine (22), which on reacting with nitrous acid at $0{ }^{\circ} \mathrm{C}$ gave the ditetrazolofuropyrimidine, 23 (Scheme 4).

\section{Conclusion}

In conclusion, we have presented various methods for the synthesis of new heteroarylfuro[2,3- $d$ ]pyrimidines such as pyrazolylfuropyrimidinethiones and condensed furopyrimidine derivatives such as furotriazolopyrimidinethiones and bis tetrazolofuropyrimidine starting from 2-amino-4,5diphenylfuran-3-carbonitrile.

\section{References}

[1]. Pang, W.; Zhu, S.; Xin, Y.; Jiang, H.; Zhu, Z. Tetrahedron 2010, 66, 12611266.

[2]. Abdel-Megid, M.; Elkazak, A. M.; Seada, M.; Farouk, O. S. J. Appl. Sci. Res. 2010, 6 (12), 2133-2138.

[3]. Maedo, Y.; Nakano, M.; Sato, H.; Migazak, Y.; Schweiker, S. L.; Smith, J. L.; Truesdale, A. T. Bioorg. Med. Chem. Lett. 2004, 14, 3907-3911.

[4]. Bhuiyan, M. M. H.; Rahman, K. M. M.; Hossain, M. K.; Rahim, M. A.; Hossain, M. I. Croat. Chem Acta. 2005, 78, 633-636.
[5]. Gangjee, A.; Zeng, Y.; McGuire, J. J.; Kisliuk, R. L. J. Med. Chem. 2005, 48, 5329-5336.

[6]. Dave, C. G.; Shah, R. D. Molecules 2002, 7, 554-565.

[7]. Gangjee, A.; Zeng, Y.; Ihnat, M.; Warnke, L. A.; Green, D. W.; Kisliuk, R. L.; Lin, F. T. Bioorg. Med. Chem. 2005, 13, 5475-5491.

[8]. Janeba, Z.; Balzarini, J.; Andrei, G.; Snoeck, R.; De Clercq, E.; Robins M. J. J. Med. Chem. 2005, 48, 4690-4696.

[9]. Robins, M. J.; Miranda, K.; Rajwanshi, V. K.; Peterson, M. A.; Andrei, G.; Snoeck, R.; De Clercq, E.; Balzarini, J. J. J. Med. Chem. 2006, 49, 391398.

[10]. Abdel-Megid, M. J. Synth. Commun. 2007, 37, 3211-3218.

[11]. Abdel-Megid, M. Pharmazie 2000, 55, 263-268.

[12]. Abdel-Megid, M.; Abbas, M.; Hassan, M. J. Heterocycl. Chem. 2007, 44, 315-322.

[13]. Ali, T. E. Eur. J. Med. Chem. 2009, 44, 4385-4392

[14]. Behalo, M. S. Phosphorus Sulfur 2009, 184 (14), 206-219.

[15]. Abu-Zied, K. H. Phosphorus Sulfur 2007, 182 (28), 447-474.

[16]. Hussein, A. H. M.; El-Gaby, M. S.; Abu-Shanab, F.; Abdel-Raheim, A. M. Org. Commun. 2009, 2(3), 66-71

[17]. Gewald, K. Chem. Ber. 1966, 99, 1002-1007.

[18]. Korbukh, I. A.; Bulychev, Y. N.; Preobrazhenskaya, M. N. Chem. Heterocyc. Compd. 1979, 15 (12), 1361-1366.

[19]. Kmetic, M.; Stanovnik, B. J. Heterocycl. Chem. 1995, 32, 1563-1565.

[20]. Abdel-Megid, M. Chem. Heterocycl. Compd. 2010, 3, 405-414.

[21]. Abdel-Megid, M. Internat. J. Chem. 2006, 16 (3), 149-157. 